\title{
RT-PCR Combined with CT Examination in the Diagnosis and Prognosis Evaluation of COVID-19 Patients in Fangcang Hospital: A Case Series
}

This article was published in the following Dove Press journal:

Journal of Multidisciplinary Healthcare

\author{
Jun-Fei Zhang ${ }^{1, *}$ \\ Jia $\mathrm{Liu}^{2, *}$ \\ Han-Ning $\mathrm{Ma}^{\prime}$ \\ Ke Feng' \\ Zhong-Wei Chen' \\ Li-Shan Yang (D) \\ Bin $\mathrm{Mei}^{3}$ \\ Jun-Jian Zhang ${ }^{4}$
}

'Department of Emergency Medicine, General Hospital of Ningxia Medical University, Yinchuan, Ningxia 750000 ,

People's Republic of China; ${ }^{2}$ Medical Experiment Center, General Hospital of Ningxia Medical University, Yinchuan, Ningxia 750000, People's Republic of China; ${ }^{3}$ Department of Social Medical Development, Zhongnan Hospital of Wuhan University, Wuhan, Hubei 43007I, People's Republic of China; ${ }^{4}$ Department of Neurology, Zhongnan Hospital of Wuhan University, Wuhan, Hubei 43007I, People's Republic of China

*These authors contributed equally to this work
Rationale: Currently, the "gold standard" is real-time reverse transcriptase-polymerase chain reaction (RT-PCR) amplification of the viral DNA for diagnosis of COVID-19 infection. However, early reports of test performance in the Wuhan outbreak showed variable sensitivities. Therefore, the simple use of RT-PCR as a discharge standard for COVID-19 patients may be risky. Early discussions suggested that $\mathrm{CT}$ should be the preferred modality for the diagnosis of COVID-19. However, the use of CT for COVID-19 discharge is controversial. In the Fangcang hospital, we performed multiple nucleic acid tests and chest CT examinations in all patients. For discharged patients, we performed multiple nucleic acid tests and chest CT scans on the basis of discharge standards to minimize the incidence of false negatives in nucleic acid tests.

Patient Concerns: Two 42-year-old male patients with mild to moderate COVID-19 were treated in the Fangcang Hospital According to the treatment, one patient was cured and discharged, while the other patient was sent to a higher-level hospital for further treatment.

Diagnoses: Real-time reverse transcriptase-polymerase chain reaction amplification of the viral DNA for diagnosis of COVID-19 infection.

Interventions: The patients received Chinese medicine and antiviral treatment in the Fangcang Hospital.

Outcomes: At follow-up, both patients were cured after treatment and returned to normal life after 2 weeks of home isolation and a negative nucleic acid test.

Lessons: The use of nucleic acid testing combined with chest CT examination can quickly diagnose patients with COVID-19 infection and evaluate their treatment in the Fangcang Hospital.

Keywords: case report, COVID-19, CT, Fangcang Hospital

\section{Introduction}

Since December 8, 2019, several cases of pneumonia with unknown cause were confirmed to be infected with a novel coronavirus, known as coronavirus disease 2019 (COVID-19), in Wuhan, Hubei province, China, which had previously not been detected in humans or animals. ${ }^{1}$ Without a therapeutic vaccine or specific antiviral drugs, early detection and isolation became essential against COVID-19. Therefore, as of February 19, the Chinese government converted 13 large-scale public places in Wuhan City, Hubei Province, into Fangcang hospitals for patients of COVID-19 with mild symptoms, and such conversion will continue to contain the spread of the COVID-19. ${ }^{2}$ From the perspective of treating patients and

Correspondence: Zhong-Wei Chen;

Li-Shan Yang

Email13995390007@163.com;

nyfyyls@।26.com
Journal of Multidisciplinary Healthcare 2021:14 |45-149

145

DovePress $\mathbf{f}$ in $\boldsymbol{\nabla}$ 


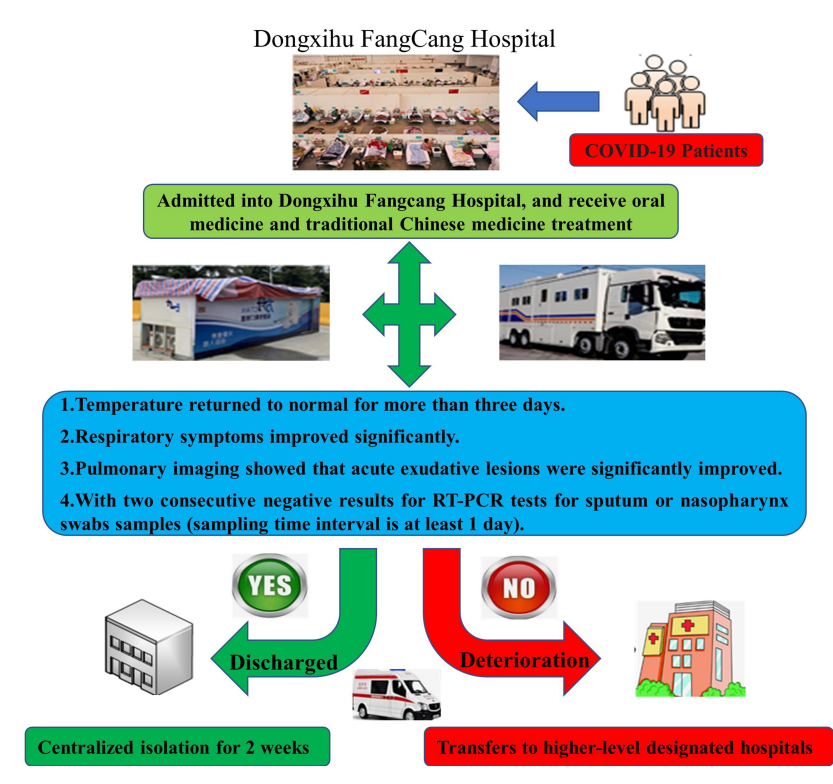

Figure I The flow chart of confirmed patients admitted into Dongxihu Fangcang Hospital.

controlling the source of infection, a completely novel model, Fangcang Hospital, emerged, which mainly received non-seriously ill patients with positive COVID19 RT-PCR tests. ${ }^{3}$ The flow chart of confirmed COVID-19 patients admitted to Fangcang Hospital is shown in Figure 1. All patients with COVID-19 were evaluated after treatment and given further admission treatment or discharge observation according to their condition (Table 1). Here, we report the different outcomes of two cases of confirmed COVID-19 patients treated in Fangcang hospitals. This study was approved by the institutional ethics committee of General Hospital of Ningxia Medical University (No. 2020-532). Because of its retrospective and emergent nature and evaluation of the imaging and clinical data of the patients, involving no potential risk, two patients obtained verbal informed consent included publication of the case details and our IRB approved the informed verbal consent process.

\section{Case Presentation Case I}

On February 15, 2020, a 42-year-old male patient, who is a businessman, presented with chief complaints of "fever for 3 days, diarrhea, anorexia, and asthenia," and a chest CT scan showed pneumonia affecting both of the lungs. On February 16, the patient was positive for COVID-19 in a nucleic acid test. The patient was admitted to the Dongxihu Fangcang hospital in Wuhan on February18. After admission, his temperature was normal, with occasional as then is and cough but no sputum. He was given oral arbidol (200 mg twice daily) and Chinese traditional medicine (COVID-19 pneumonia No. 2, once a day), which has shown promise in treating COVID-19 patients according to the National Administration of Traditional Chinese Medicine. The patient's symptoms decreased. The patients underwent chest CT scans on February 20, 28, and March 5, respectively, and the results showed that the inflammation in pneumonia gradually improved. The patient's COVID-19 pneumonia nucleic acid test was positive on February 20 and 28, but was negative on March 1 and 5, and met the other criteria for discharge by March 6 . The patient was discharged on March 7 (Figure 2).

\section{Case 2}

On February 12, 2020, a 42-year-old male patient, who was ordinarily a resident in Wuhan, presented with chief complaints of "fever for 1 week, cough, and asthenia," and she was admitted to Dongxihu Fangcang hospital. She was administered oral arbidol (200 mg twice daily) and Chinese traditional medicine (COVID-19 pneumonia No. 2, once a day; LianhuaQingwen Capsule ( 4 capsules at a time, 3 times

Table I Clinical Outcome Criteria for All COVID-19 Patients

\begin{tabular}{|l|l|}
\hline Outcome & Specific Terms \\
\hline Deterioration* & $\begin{array}{l}\text { I. Symptoms persisted or worsened for more than } 7 \text { days. } \\
\text { 2. At rest, respiratory rate } \geq 30 \text { bpm or blood oxygen saturation } \leq 93 \% . \\
\text { 3. Pulmonary imaging showed that the lesions progressed more than } 50 \% \text { within } 48 \text { hours. }\end{array}$ \\
\hline Discharge & $\begin{array}{l}\text { I. Temperature returned to normal for more than } 3 \text { days. } \\
\text { 2. Respiratory symptoms improved significantly. } \\
\text { 3. Pulmonary imaging showed that acute exudative lesions were significantly improved. } \\
\text { 4. With two consecutive negative results for RT-PCR tests for sputum or nasopharynx swabs samples (sampling time interval is at } \\
\text { least I day). }\end{array}$ \\
\hline
\end{tabular}

Notes: *Patients with any one of the following conditions were defined as deterioration of COVID-19. 


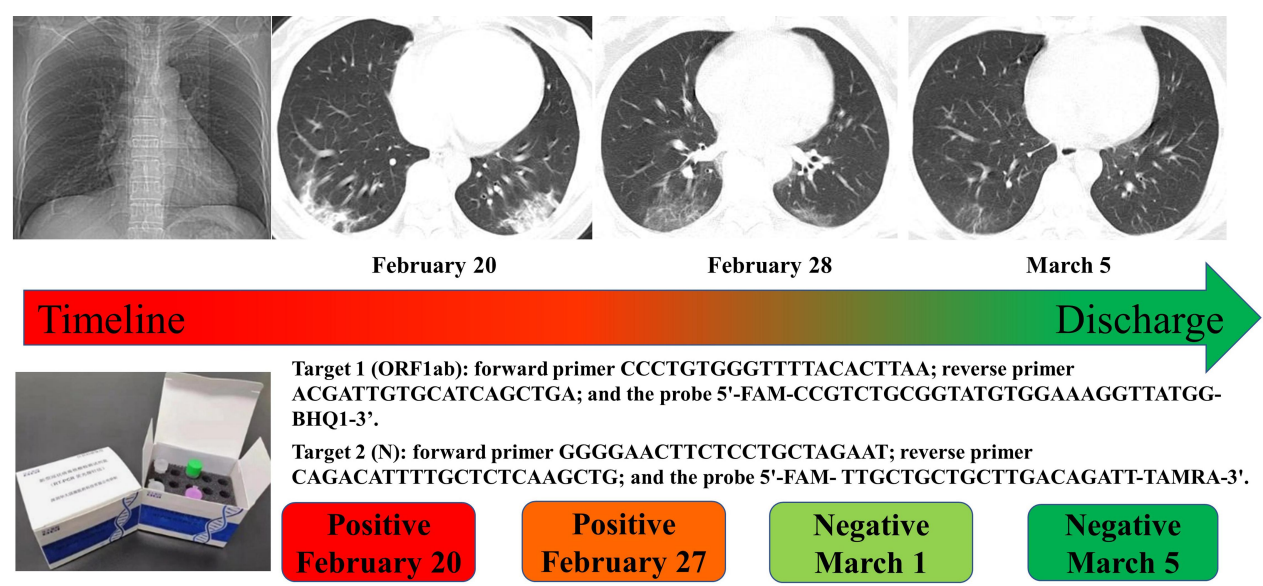

Figure 2 Case I: Results of the patient's COVID-19 pneumonia nucleic acid detection and chest CT results.

a day). The patient's temperature remained normal 4 days after admission, with continued cough without sputum. On February 14, the patient's first COVID-19 nucleic acid test was positive, and a chest CT scan on the next day showed bilateral lung inflammation. The patient developed fever and cough again on February 20. In the nucleic acid tests, she was positive on February 22 and 24, respectively. Meanwhile, the patient underwent chest CT scans on February 21 and 24, respectively, and the results showed that the inflammation in pneumonia gradually aggravated. She was transferred to a traditional hospital designated for more intensive COVID19 care on February 25 to continue treatment (Figure 3).

\section{Discussion}

Fangcang hospitals were developed and used for the first time in China to tackle the COVID-19 outbreak. ${ }^{4}$ They improved the distribution of patients with COVID-19 according to the severity of their symptoms, increasing the overall efficiency of care. At present, research reports on the clinical characteristics of patients with COVID-19 have mainly focused on inpatients or critically ill patients. ${ }^{5}$ These studies provide an important reference for formulating COVID-19 pneumonia diagnosis and treatment guidelines. ${ }^{4}$ The Fangcang Hospital mainly treats patients with mild to moderate COVID-19. There are few reports on the clinical characteristics of these patients, who are the main component of COVID-19. ${ }^{3}$ Most patients have mild manifestations and an excellent prognosis. The elderly and patients with underlying comorbidities are prone to develop severe conditions. Male sex, older age, chronic medical illness, epidemiological history, and signs and symptoms were all risk factors for mild to moderate COVID-19 patients who were seriously ill and needed to be transferred to higher-leveldesignated hospitals for treatment.

In our report, two patients with mild to moderate COVID-19 showed different treatment outcomes after
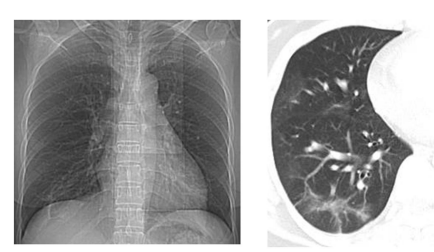

February15

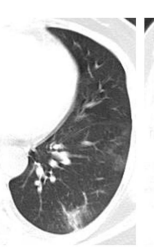

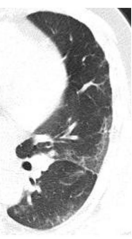

February 21

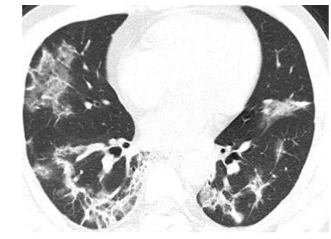

February 24

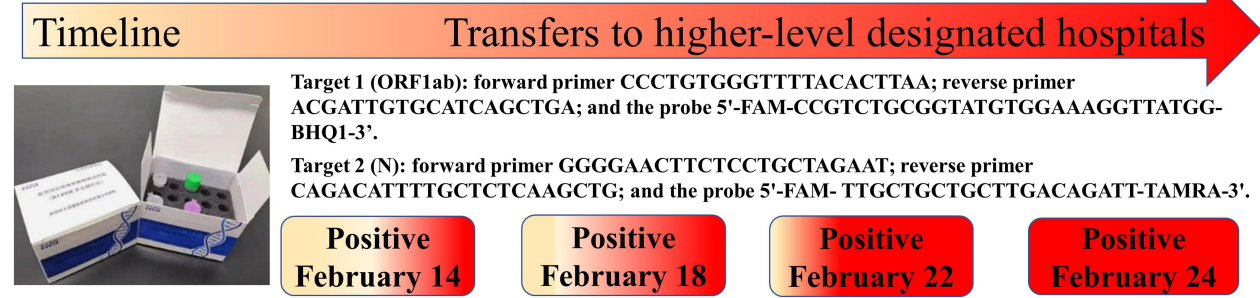

Figure 3 Case 2: Results of the patient's COVID-19 pneumonia nucleic acid detection and chest CT. 
treatment in the Fangcang hospital. Case 1 received Chinese medicine and antiviral treatment in the Fangcang hospital, and met the discharge standards after passing the continuous COVID-19 nucleic acid virus test and chest CT scan. After being discharged from the hospital, case 1 was isolated at home for 14 days. After the nucleic acid test was negative again, the patient returned to normal life. Case 2 was also treated with traditional Chinese medicine and antiviral drugs in the Fangcang hospital, but the patients were all positive through the continuous COVID-19 nucleic acid virus test. At the same time, the chest CT scan showed that the patient's lung inflammation gradually increased. Case 2 was sent to a designated hospital for further treatment. Follow-up showed that the patient recovered after 2 weeks of treatment in the hospital and returned to normal life after a negative nucleic acid test after further home isolation for 2 weeks.

Currently, the "gold standard" is real-time reverse transcriptase-polymerase chain reaction (RT-PCR) amplification of the viral DNA for diagnosis of COVID-19 infection. However, RT-PCR may produce initial false-negative results. Long et al suggested that patients with typical CT findings but negative rRT-PCR results should be isolated, and rRT-PCR should be repeated to avoid misdiagnosis. ${ }^{7}$ Medical imaging, as a routine imaging tool for pneumonia diagnosis, is relatively easy to perform and can produce a fast diagnosis. The imaging of coronavirus disease (COVID-19) can be sorted into four phases: early phase, progressive phase, severe phase, and dissipative phase. The spectrum of CT findings of each phase can indicate the temporal progression of the disease. ${ }^{8}$ In this context, chest $\mathrm{CT}$ is of great significance in the detection and surveillance of COVID-19infection. ${ }^{6}$ The chest computed tomography findings can show the imaging characteristics of confirmed cases of COVID-19 pneumonia. ${ }^{9}$ Early discussions suggested that CT should be the preferred modality for the diagnosis of COVID-19. However, pneumonia caused by COVID-19 shared overlapping imaging manifestations with other types of pneumonia. So the use of CT for COVID-19 discharge is controversial. Other decisions to guide the detection and diagnosis of COVID-19 virusinduced pneumonia in clinical practice should also be developed, such as the proposed quantitative scoring criteria. ${ }^{10} \mathrm{CT}$ scans of COVID-19-infected patients confirmed by RT-PCR showed that the surrounding lungs and ground-glass opacity developed rapidly, which proved that the results of RT-PCR and CT can be judged mutually. ${ }^{11}$ In the Fangcang hospital, we performed multiple nucleic acid tests and chest CT examinations in all patients. For discharged patients, we performed multiple nucleic acid tests and chest $\mathrm{CT}$ scans on the basis of discharge standards to minimize the incidence of false negatives in nucleic acid tests. Our two cases also show that it is a double insurance for patients with COVID-19 infection through nucleic acid testing combined with chest $\mathrm{CT}$ to evaluate the treatment status.

In summary, the use of nucleic acid testing combined with chest CT examination can quickly diagnose patients with COVID-19 infection and evaluate their treatment, especially for screening patients with large-scale infections, which is a practical solution.

\section{Data Access Statement}

The original data are part of the electronic health records of the Zhongnan Hospital of Wuhan University, Wuhan, China. Therefore, data are not accessible for the public. Any questions about the analyzed data can be addressed to the corresponding author (Zhong-Wei Chen). Requests for materials should be addressed to Zhong-Wei Chen (Email: 13995390007@163. com) and Li-Shan Yang (Email: nyfyyls@126.com).

\section{Acknowledgments}

We thank all the patients included in this study.

\section{Funding}

This study was supported by the Natural Science Fund of Ningxia (2020AAC02003) and the Ningxia Medical University (XE202002).

\section{Disclosure}

All of the authors declare that they have no conflicts of interest for this work.

\section{References}

1. Chen S, Yang J, Yang W, et al. COVID-19 control in China during mass population movements at new year. Lancet. 2020;395 (10226):764-766. doi:10.1016/s0140-6736(20)30421-9

2. Chen N, Zhou M, Dong X, et al. Epidemiological and clinical characteristics of 99 cases of 2019 novel coronavirus pneumonia in Wuhan, China: a descriptive study. Lancet. 2020;395 (10223):507-513. doi:10.1016/S0140-6736(20)30211-7

3. Lu H, Stratton CW, Tang YW. Outbreak of pneumonia of unknown etiology in Wuhan, China: the mystery and the miracle. J Med Virol. 2020;92(4):401-402. doi:10.1002/jmv.25678

4. Chen C, Zhao B. Makeshift hospitals for COVID-19 patients: where health-care workers and patients need sufficient ventilation for more protection. J Hosp Infect. 2020;105(1):98-99. doi:10.1016/j. jhin.2020.03.008 
5. Wang X, Fang J, Zhu Y, et al. Clinical characteristics of non-critically ill patients with novel coronavirus infection (COVID-19) in a Fangcang Hospital. Clin Microbiol Infect. 2020;26(8):1063-1068. doi:10.1016/j.cmi.2020.03.032

6. Ai T, Yang Z, Hou H, et al. Correlation of chest CT and RT-PCR testing for Coronavirus Disease 2019 (COVID-19) in China: a report of 1014 cases. Radiology. 2020;296(2):E32-E40. doi:10.1148/ radiol.2020200642

7. Long CQ, Xu HX, Shen QL, et al. Diagnosis of the Coronavirus disease (COVID-19): rRT-PCR or CT? Eur $J$ Radiol. 2020;126:108961. doi:10.1016/j.ejrad.2020.108961

8. Li M, Lei P, Zeng B, et al. Coronavirus Disease (COVID-19): spectrum of CT findings and temporal progression of the disease. Acad Radiol. 2020. doi:10.1016/j.acra.2020.03.003
9. Lin X, Gong Z, Xiao Z, et al. Novel coronavirus pneumonia outbreak in 2019: computed tomographic findings in two cases. Korean J Radiol. 2020;21(3):365-368. doi:10.3348/kjr.2020.0078

10. Li Z, Zeng B, Lei P, et al. Differentiating pneumonia with and without COVID-19 using chest CT images: from qualitative to quantitative. J Xray Sci Technol. 2020. doi:10.3233/XST-200689

11. Wei J, Xu H, Xiong J, et al. 2019 novel Coronavirus (COVID-19) pneumonia: serial computed tomography findings. Korean J Radiol. 2020;21(4):501-504. doi:10.3348/kjr.2020.0112

\section{Publish your work in this journal}

The Journal of Multidisciplinary Healthcare is an international, peerreviewed open-access journal that aims to represent and publish research in healthcare areas delivered by practitioners of different disciplines. This includes studies and reviews conducted by multidisciplinary teams as well as research which evaluates the results or conduct of such teams or healthcare processes in general. The journal covers a very wide range of areas and welcomes submissions from practitioners at all levels, from all over the world. The manuscript management system is completely online and includes a very quick and fair peer-review system. Visit http://www.dovepress.com/testimonials. php to read real quotes from published authors. 